\title{
The effectiveness of mirror therapy with repetitions on lower extremity motor recovery, balance and mobility in patients with stroke
}

\author{
Kritika Verma, Jaspreet Kaur, Manoj Malik, Neerja Thukral \\ Department of Physiotherapy, Guru Jambheshwar University of Science and Technology, Hisar, \\ Haryana, India
}

\begin{abstract}
Objective. To evaluate the effectiveness of mirror therapy using motor imagery training on lower extremity motor functioning, balance and mobility in the patients after stroke.

Material and methods. A randomized, controlled trial (RCT). Rehabilitation institutes and hospitals. 56 patients with stroke were adventitiously assigned into two different groups i.e., "Group A" (experimental group) and "Group B" (control group). The mean age of Group-A was $50.67 \pm 11.18$ years and Group-B was $49.16 \pm 10.28$ years without volitional ankle dorsiflexion. The post-stroke patients under experimental group received the comprehensive rehabilitation therapy along with the MT for LE. Participants received MT near 30 minutes in a day, 6 days a week and continued for up-to 6 weeks. Whereas, the post-stroke patients under control group received the placebo therapy along with comprehensive rehabilitation therapy. For motor recovery Brunnstrom stages were used, to assess spasticity the Modified Ashworth Scale was used, for functional ability the Functional Ambulation Category was used and balance was assessed by Berg Balance Scale.

Results. The experimental group after intervention showed remarkable changes in FAC (mean difference $=0.79$, $95 \% \mathrm{Cl}=0.65-1.26, \mathrm{p}=0.000$ ) and BBS (mean difference $=3.10,95 \% \mathrm{Cl}=3.21-4.88, \mathrm{p}=0.000$ ) as contrasted to the control group.

Conclusions. MT along with the conventional therapy is more effective to improve the balance, mobility and motor recovery as compared to only conventional rehabilitation therapy.
\end{abstract}

Keywords: mirror therapy (MT), motor imagery training, lower extremity (LE), randomized controlled trial $(\mathrm{RCT})$, rehabilitation, motor recovery, spasticity, functional ability, balance

\section{INTRODUCTION}

Stroke can be defined as the "immediate loss of the neurological functions of a person caused due to an obtrusion of the blood flow to the brain". It causes hindrance of various brain functions resulting in a variety of symptoms [1] like abnormal muscle activation, alterations in movement of the selective joints, tone, balance and coordination [2]. The life of patient is highly influenced by coordination, voluntary \& involuntary movements and the gait pattern which is being regulated by cerebellum. Consequently, in cerebrovascularlacci- dents, an individual encounters a dropping of voluntary control and is unable to carry out various activities of LE [2] as well as upper limb. It mainly gives rise to the half body paralysis, accompanied by sensory $\&$ motor loss. In addition, changes in the level of consciousness; cognitive, perceptual and speech disturbances; and sometimes the visual defects, anxiety and depression also occurs [1]. It is thus a major cause of impaired activities of daily living, often leadingLto dependency [3] or long term care [4,5]. Motor functions of lower extremity are often impaired along with the impairment of gait pattern [2,6], balance [3], mobility [7] in the 
post-stroke patient. Stroke is one of the leading causes of mortality and disability [8]. According to the study of 2013, Jeyaraj Durai Pandian and Paulin Sudhan proposed that in India, approximate prevalence rate of stroke lies between 84 to 262 per 100,000 in underdeveloped areas and 334 to 424 per 100,000 in developed areas [8].

Physical therapy treatments include balance training, neuromuscular re-training, pre-walking functional chores for instance, weight shifts in deskbound or erect positions, transfer events [7] and independent posture maintenance etc for rehabilitation of stroke patient. Additionally, neurofacilitation approaches and functional electrical stimulation (FES) along with the muscle strength training are also given to the patient. Moreover, robot-aided sensorimotor stimulation is a method of treatment which is also used to enhance motor performance and improve functional outcome [9]. Nevertheless, primary focus should be on repetitive tasks, specific or goal oriented practice [10] intensity of treatment should be high with a feedback mechanism on performance [11]. The treatment strategies which are also been used from last few years consist of Mental practice; Constraint induced movement therapy and Mirror Therapy.

Mirror therapy (MT) is a comparatively new therapeutic intervention of its kind with its primary focus on moving the impaired limb [11]. Ease of usage of MT and its low cost might account to an appropriate alternative [6]. Ramachandran and Roger-Ramachandran initially introduced this kind of therapy to treat phantom limb pain after amputation [12]. Altschuler et al. suggested that significant improvements were seen in the patients treated with MT than without MT [13]. Whereas, Striven and Stoykov also found significant improvements in stroke patients who undertook MT for 3 to 4 weeks, as marked increase in Fugl-Meyer Assessment scores, movement speed, active range of motion(ROM) and hand agility were obtained $[14,15]$. Correspondingly, remarkable improvement of grasping strength and hand movement of the impaired arm after 2 weeks of intense MT on chronic stroke patients were recorded by the Sathian et al. [16].

The mirror therapy works on the principle of neuroplasticity which is accentuated by the visual cues and mirror neuron system [17]. It works by allowing the patient to observe the reflection of the unaffected limb sited as the affected limb by looking in the mirror. Perception of the affected limb and motor performance can be therapeutically improved by strong visual indication from the mirror [11]. A current systematic review of imaging studies partially supports this hypothesis about the affect of mirror therapy on the brain [18] as it specifies that motor activities are promoted contralateral to the affected side by using the mirror therapy [17]. The main focus of the Mirror Therapy is to activate the mirror neurons in the brain; these can also be activated by seeing others performing the same activities. By using this idea we can improve a patient's voluntary movements, balance, mobility, bodily functions, gait pattern, reduce synergic pattern and also improve coordination due to which a patient's daily living activities are improved along with better quality of life. This study is hence designed to emphasize on mirror neuron activation and to determine the effectiveness of MT with repetitions on LE motor recovery, balance, and mobility in patients with stroke.

\section{MATERIAL AND METHODS}

\section{Participants}

56 patients who had stroke were randomly assigned into two different groups: Group A (experimental group) and Group B (control group). The mean age of Group A was $50.67 \pm 11.18$ years and Group B was $49.16 \pm 10.28$ years without volitional ankle dorsiflexion.

The possible participants were appropriately assigned with the following inclusion criteria: post-stroke hemiplegic that affected lower extremity (LE) function and performance; adults with age over 30 years in all the stages of stroke; able to understand and follow the simple verbal commands; non-critical cognitive disorders that might disturb the study design; the patient's medical condition should be stable and the patient must have been ambulatory before stroke.

If the participants manifested any of the following elements, then from the study they were excluded: patients with neglect, pusher syndrome; visual deficits; any other associated neurological disorder, psychological or emotional problems; cardiovascular instability; any musculoskeletal 
disorder affecting mobility and post-operative in lower extremity; range of motion should not be limited in the lower extremity.

All the patients were informed about the study and a written consent was obtained from them.

\section{Study design}

This is a randomized controlled trial (RCT). T trial design was guided in accordance the Consolidated Standards of Reporting Trials (CONSORT) statement (figure 1) [19]. The trial has been reported honoring to the Standard Protocol Items: Recommendations for Interventional Trials (SPIRIT) guidelines [20].

\section{Approval and consent form institutional ethics committee (IEC)}

The approval of the present study was taken from the Institutional Ethics Committee as per the guidelines assigned by Indian council of Medical Research and the study protocol was approved by IEC, Guru Jambheshwar University of Science \&Technology, Hisar vide letter number PTY/2019/1014 dated on 11/9/2019. The CTRI registration number was CTRI/2020/03/023773. The patients who were interested to participate in the study were explained about the procedure prior to participate and a written consent was obtained.

\section{Sample size}

The sample size was calculated with the formula: $n_{i=} 2\left(Z_{\alpha}+Z_{(1-\beta)}\right)^{2} \sigma^{2} /\left(\mu_{s}-\mu_{t}-\delta\right)^{2}$, where:

- $\mathrm{n}_{\mathrm{i}}=$ sample size required in each group

- $\sigma=$ standard deviation

- $\delta=$ minimal clinically important difference (MCID)

- $\mathrm{Z} \alpha=1.96$

- $\mathrm{Z} \beta=0.8416$

- $\mu \mathrm{S}-\mu \mathrm{t}=1$

By putting all the required values to the formula, the sample size received was 56 including dropout rate of $5 \%$ with age in between 30 and 60 years. Thereafter, participants were randomly assigned into two groups.

\section{Randomisation}

The patients were allocated to different groups by simple random sampling. The randomisation was done using chit method. Subjects were asked to pick up a chit from a bowl on which an alphabet was written like ' $\mathrm{A}$ ' and ' $\mathrm{B}$ '. Then accordingly

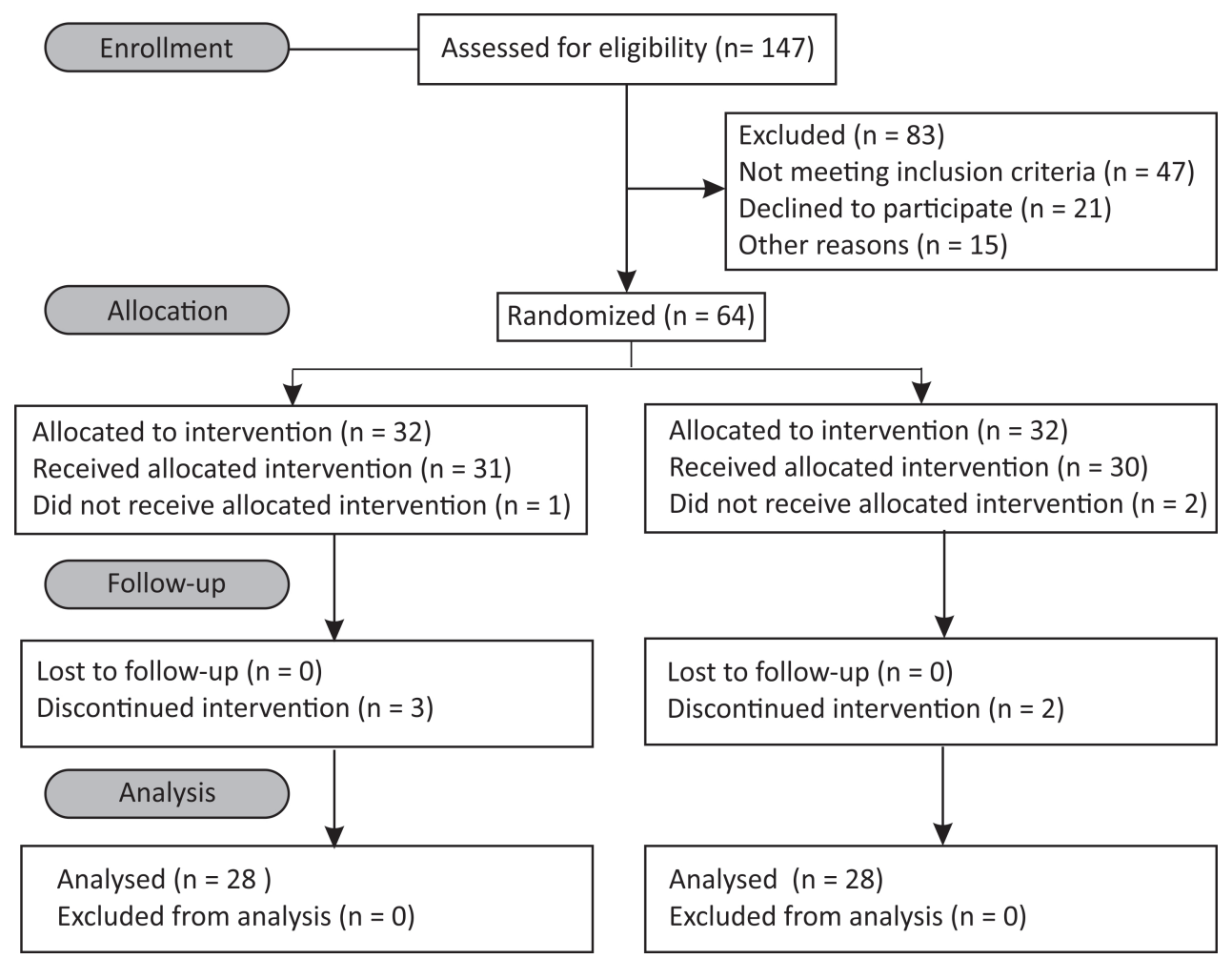

FIGURE 1. The CONSORT (Consolidated Standards of Reporting Trials) 2010 flow diagram for the study 
subjects were assigned into two groups Group A and Group B which were experimental group and control group respectively. Subjects under experimental group received stroke intervention with mirror therapy. Whereas, subjects under control group received stroke intervention without mirror therapy.

\section{Intervention}

The patients were divided randomly into two groups - Group A (experimental group) and Group B (control group). The patients in Group A received the comprehensive rehabilitation therapy along with the mirror therapy for lower extremity. In 1 st week, normal range of motion (ROM) exercises was started with unaffected leg being overlooked into the mirror hiding the affected leg. Meanwhile, patient was asked to imitate same exercises with the affected leg as well. Once the patient started doing it, the difficulty level was increased and other factors like balance and gait pattern were also taken into consideration. From 2nd week onwards, patient's balance was worked upon, initially started with sit to stand, then gradually increased to other balance exercises like standing from wide base of support (BOS) to narrow BOS, perturbations in standing, catch and throw, raise one leg up and swiss ball exercises as well. All the exercises were performed in front of the mirror. Variations were made in the protocol according to the patient's ability, improvements, stamina, desire and will. Along with it, the gait training was also started for the patient in front of the mirror. The patient was made to walk in front of mirror by using a parallel bar walk way, so that the patient could see his/her walking pattern in front of the mirror and if wrong pattern was followed, it could be fixed and walking was commenced again by minimizing faults. An additional 30 minutes a day were given to the patients of Group A. Participants received MT near 30 minutes in a day, 6 days a week and continued for upto 6 weeks. Meanwhile, the stroke patients under control group underwent the placebo therapy and comprehensive rehabilitation therapy in which the treatment protocol was programmed according to the patient assessment, current condition and contained the neuro-developmental facilitation techniques, physical therapy, occupational therapy and speech therapy (as per the requirement).

\section{Outcome measures}

Outcomes were measured on the respect of motor recovery (Brunnstrom stages), spasticity (Modified Ashworth Scale), functional ability (Functional Ambulation Category) and balance (Berg Balance Scale). The measures were taken a day before the intervention to be started, at the $3^{\text {rd }}$ week and at the $6^{\text {th }}$ week of treatment.

\section{Motor recovery}

The Brunnstrom stages were used to assess motor recovery. It sets out a sequence of stages of recovery from hemiplegia after stroke to the normal isolated movements. The Brunnstrom Approach follows six proposed stages of sequential motor recovery after stroke which are (1) completely flaccid; (2) spasticity appears and basic limb synergy develops; (3) remarkable spasticity; (4) spasticity begins to decline and patient is able to move with less resistance; (5) spasticity declines and the patient can do isolated joint movements and (6) no longer spasticity, near normal movements and coordination.

\section{Spasticity}

Modified Ashworth Scale (MAS) was used as a simple measure of spasticity to evaluate the muscle tone and it measures resistance during passive soft-tissue stretching. Scoring consists of 6 points which starts from 0 i.e., no increase in muscle tone and ends up at 4 in which the affected part becomes rigid in flexion or extension [1].

\section{Functional ability}

The Functional Ambulation Categories (FAC) is a functional walking test that estimates ambulation ability. This 6-point scale evaluates ambulation status by influencing how often reinforcement is required when walking, nevertheless of whether or not they utilize a particular assistive device [21]. The FAC does not appraise endurance, as the patient is only recommended to walk roughly $10 \mathrm{ft}$. It consists of 6 points which represents the functional ability of the patient from non functional ambulation to the independent ambulation with independent stair climbing.

\section{Balance}

Berg Balance Scale (BBS) is used to assess the balance. There are 14 functional performance test 
which starts from maintaining the balance by doing sit to stand to noticeable increase in balance up to stand on a single leg. Every step consists of 0 to 4 possibilities, through which they got points, the total points are 56 . The points more than 40 represents a good balance of the patient, from 20 to 40 it shows the moderate balance and below 20 means the patient's balance is very poor [1].

\section{Data analysis}

Data was analysed using SPSS software. The patients, who completed the entire therapy course, were included into the analysis. Whereas, the patients who dropped out or were unable for the follow up sessions, the purpose to treat became scrappy and the analysis was not possible. Normality of data was checked by using Shapiro-Wilk test. Independent t-test was used to compare the differences in baseline characteristic between the experimental and control group in advance of the therapy. Before and after the treatment, paired sample t-test was used to differentiate FAC and BBS within each group. Finally, Independent t-test was used to study Differences of pre and post test changes in FAC and BBS between the experimental and the control group. Selected significance level for this analysis was $\mathrm{p}=0.05$.

\section{RESULTS}

The participants who volunteered for the study were enrolled from the MSS Hospital Pitampura, New Delhi. A total of 147 patients were screened to participate in the study. 47 were omitted as they were unable to meet the inclusion criteria, 21 subjects denied their participation in study and 15 subjects were excluded due to other reasons. All through the enrollment period inclusion criteria were met by 64 subjects, out of whom half were assigned to the MT group and other half to the control group randomly. 8 more subjects withdrew their participation. The protocol was completed by 56 subjects finally.

The mean age of MT group was 50.67 \pm 11.18 years old and that of control group was $49.16 \pm$ 10.28 years old with $43.79 \pm 33.02$ months being the mean time for getting rehabilitation after the Stroke. Baseline characteristics were evaluated/ measured a day before starting of intervention and were found to be same for both the groups $(p>0.05)$. The characteristics are shown in Table 1.

Primary outcome measures FAC (mean difference $=0.79,95 \% \mathrm{CI}=0.65-1.26, \mathrm{p}=0.000)$ and BBS (mean difference $=3.10,95 \% \mathrm{CI}=3.21-4.88$, $\mathrm{p}=0.000$ ) improved significantly more in the intervention group but there was no significant improvement in control group post-intervention $($ FAC $(p=0.083)$ and BBS $(p=0.017)$ (Table 2, Figure 2).

\section{DISCUSSION}

The purpose of the study was to assess the efficacy of MT with repetitions on LE to improve the motor recovery, balance, functional mobility and reduction of gait deviation in patients with stroke. In the present study the Functional Ambulation Category (FAC) and Berg Balance Scale (BBS)

TABLE 1. Baseline characteristics of both groups

\begin{tabular}{|c|c|c|c|c|}
\hline \multicolumn{2}{|l|}{ Variables } & \multirow{2}{*}{\begin{tabular}{|c|} 
Experimental group \\
$50.67 \pm 11.18$ \\
\end{tabular}} & \multirow{2}{*}{\begin{tabular}{|c|} 
Control croup \\
$49.16 \pm 10.28$ \\
\end{tabular}} & \multirow{2}{*}{$\begin{array}{l}\text { p value } \\
0.661\end{array}$} \\
\hline Age & mean $\pm S D$ & & & \\
\hline & Range & 38 & 34 & \\
\hline \multirow[t]{2}{*}{ Gender \% } & Male & 0.8095 & 0.6842 & \multirow[t]{2}{*}{0.374} \\
\hline & Female & 0.1905 & 0.3158 & \\
\hline Duration of stroke till pre-intervention & mean $\pm S D$ & $26.71 \pm 12.67$ & $24.75 \pm 11.57$ & 0.613 \\
\hline \multirow[t]{2}{*}{ Side affected } & Right & 0.5714 & 0.4211 & \multirow[t]{2}{*}{0.355} \\
\hline & Left & 0.4286 & 0.5789 & \\
\hline \multirow[t]{2}{*}{ Pre-intervention data for FAC } & mean $\pm S D$ & $3.62 \pm 1.01$ & $3.05 \pm 1.08$ & \multirow[t]{2}{*}{$0.025^{*}$} \\
\hline & Range & 4 & 4 & \\
\hline \multirow[t]{2}{*}{ Pre-intervention data for BBS } & mean $\pm S D$ & $44.52 \pm 10.16$ & $43.37 \pm 9.55$ & \multirow[t]{2}{*}{0.925} \\
\hline & Range & 32 & 30 & \\
\hline
\end{tabular}

SD -standard deviation; * - statistically significant; FAC - functional ambulation category; BBS - Berg Balance Scale 
TABLE 2. Within group comparison using paired t test

\begin{tabular}{|c|c|c|c|c|c|c|c|}
\hline \multirow[t]{2}{*}{ Outcome } & \multirow[t]{2}{*}{ Group } & \multirow{2}{*}{$\begin{array}{l}\text { Pre-intervention } \\
\text { mean } \pm S D\end{array}$} & \multirow{2}{*}{$\begin{array}{l}\text { Post- intervention } \\
\text { mean } \pm S D\end{array}$} & \multicolumn{2}{|c|}{ 95\% confidence interval } & \multirow[t]{2}{*}{$t$ value } & \multirow[t]{2}{*}{$p$ value } \\
\hline & & & & Lower & Upper & & \\
\hline \multirow[t]{2}{*}{ FAC } & Experimental group & $3.62 \pm 1.01$ & $4.19 \pm 0.67$ & 0.64784 & 1.25693 & 6.523 & 0.000 \\
\hline & Control group & $3.05 \pm 1.08$ & $3.21 \pm 0.37$ & -0.02267 & 0.33846 & 1.837 & 0.083 \\
\hline \multirow[t]{2}{*}{ BBS } & Experimental group & $44.52 \pm 10.16$ & $47.05 \pm 1.83$ & 3.21477 & 4.88047 & 10.138 & 0.000 \\
\hline & Control group & $43.37 \pm 9.55$ & $43.95 \pm 0.96$ & 0.11565 & 1.04225 & 2.625 & 0.017 \\
\hline
\end{tabular}

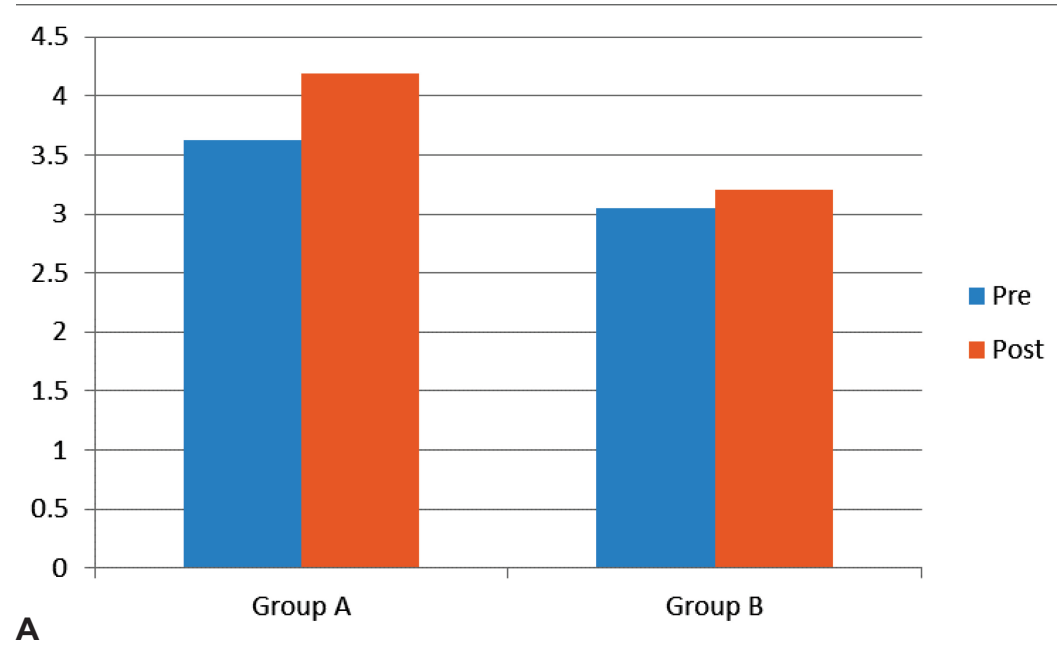

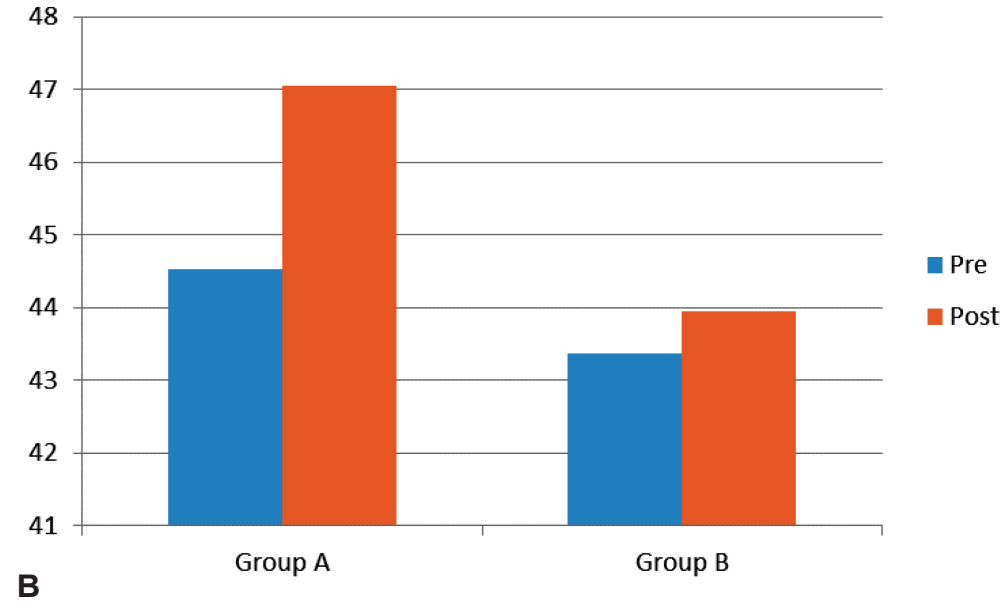

FIGURE 2A,B. Represented the mean values of the patient groups pre and post interventions under group $A$ (experimental group) and group $B$ (control group) by using the FAC and BBS. (A) represents the changes in pre and post intervention using $F A C$ in both the groups whereas (B) represents the changes in BBS. The experimental group demonstrates the significant increase in the post intervention by using both the FAC as well as in BBS as compared to the control group post intervention. expressed the significant favourable results to the study after using the mirror therapy intervention. We incorporated a randomised controlled trial with a total number of 69 patients from which the 64 patients were included in the study and only 28 patients in each group completed it.

In correspondence to the previous studies, the standard MT had a particular effect on motor functionality, walking velocity, capacity of balancing, PROM for ankle dorsiflexion and step length as compared to the control group interventions [4]. The result is dependent upon the protocol selected for the MT and its underlying neural mechanism [22]. Particularly, the flexor synergy patterns were improved during post intervention as the hip-knee flexion and ankle dorsiflexion activities were performed in the protocol. The extensor synergy did not show any favorable alterations, as these synergistic elements were not included into the protocol. The flexion movements of knee and ankle might have activated the flexor reaction during follow up protocol while performing the activities in sitting and standing position. Approximately all the activities involved in the protocol enhances the FMA-LE coordination items. The motor components were stimulated to the unaffected side so as to accelerate the corresponding movements of the affected side [2]. Wu et al. found that there was a 
considerable enhancement in movement performance after MT intervention as collated to the CT intervention, by using the FMA measurements [10]. MT perhaps provided the proper visual input or might be compensating the absent or diminished proprioceptive inputs from the affected side of the body [23]. Cortical reorganization is the reason for the positive results [24].

Some of the studies were based on the movements performed by the lower extremity in MT intervention and conclude the result. Crosby, Marrocco et al. deployed hip flexion and extension complete hip extension was not feasible [25]. Lee et al. provided three stage training in their MT protocol, which were: knee extensor and ankle dorsi-flexor, knee flexor and ankle dorsi-flexor, and hip-knee flexors and ankle dorsi flexors [26]. It improves the significant gait movements in the MT group. However, it does not figure out a specific motor component in the affected limb. Mohan et al. framed out the hip abduction and external rotation and the movements to its reversal [3]. Similarly, Sütbeyaz et al. worked on only one movement and that was ankle dorsiflexion for MT [7]. Homogeneously to the present study all the previous studies performed MT for the 30 minutes $[3,7,25]$.

The present RCT reflects that the rehabilitation protocol of the stroke patient is more effective with the MT as compared to the intervention without using MT. Functional Ambulation Category (FAC) and Berg Balance Scale (BBS) were used to measure balance and functional activities of the patients. This study showed that there were significant improvements in FAC as well as BBS which reflects that there were visible positive changes in the balance and functional activities of the patient. movements in long sitting position, whereas the

It might not be possible to see the results in initial weeks, but the MT group received continuous therapy for 30 minutes daily, 6 days a week for 6 weeks which showed the comparable good results as compared to the control group.

The limitations of this study include deprivation of long term follow-up cases, the hip extension exercises could not be performed in front of the mirror due to the position hindrance. If there was any misinterpretation related to the optical illusion, there was no objective tool to measure the extent of illusion. If there was any cardiac instability in the patients, physical interventions could not be carried out thoroughly as it would have caused further damage to the body. Future study may consider the effectiveness of prolonged duration of MT performing on lower extremity with task oriented activities. The duration of the intervention of MT can be predicted so that the great results could be found out. A proper protocol can be programmed which will include different activities considering the equipments like swiss ball, treadmill etc and its effectiveness with respect of duration can be found out.

\section{CONCLUSIONS}

Using MT for stroke patients along with the conventional therapy exhibits significant improvements in balance, mobility and motor recovery; which were measured by the FAC and BBS. The FAC and BBS showed a substantial positive result in the experimental group (MT group) contrary to the control group. Thus, MT can be used as an adjunct therapy in addition to conventional therapies for improving motor performance, balance and mobility in chronic stroke patients.

Conflict of interest: none declared Financial support: none declared

\section{REFERENCES}

1. Sullivan SBO, Schmitz TJ. Physical rehabilitation. F.A. Davis Company, 2006.

2. Arya KN, Pandian S, Kumar V. Effect of activity-based mirror therapy on lower limb motor-recovery and gait in stroke: A randomised controlled trial. Neuropsychol Rehabil. 2019;29(8):1193-210.

3. Mohan U, Babu SK, Kumar KV, Suresh BV, Misri ZK, Chakrapani M. Effectiveness of mirror therapy on lower extremity motor recovery, balance and mobility in patients with acute stroke: A randomized sham-controlled pilot trial. Ann Indian Acad Neurol. 2013; 16(4):634-9.

4. Broderick P, Horgan F, Blake C, Ehrensberger M, Simpson D, Monaghan K. Mirror therapy for improving lower limb motor function and mobility after stroke: A systematic review and meta-analysis. Gait Posture. 2018 Jun;63:208-220.

5. Hung GKN, Li CTL, Yiu AM, Fong KNK. Systematic Review: Effectiveness of Mirror Therapy for Lower Extremity Post-Stroke. Hong Kong J Occup Ther. 2015;26(2015):51-9.

6. Ji SG, Kim MK. The effects of mirror therapy on the gait of subacute stroke patients: A randomized controlled trial. Clin Rehabil. 2014;29(4):348-54.

7. Sütbeyaz S, Yavuzer G, Sezer N, Koseoglu BF. Mirror Therapy Enhances Lower-Extremity Motor Recovery and Motor Functioning After Stroke: A Randomized Controlled Trial. Arch Phys Med Rehabil. 2007;88(5):555-9. 
8. Pandian JD, Sudhan P. Stroke Epidemiology and Stroke Care Services in India. J Stroke. 2013;15(3):128.

9. Volpe BT, Krebs HI, Hogan N, Edelstein OTR L, Diels C, Aisen M. A novel approach to stroke rehabilitation: robot-aided sensorimotor stimulation. Neurology. 2000 May 23;54(10):1938-44.

10. Wu CY, Huang PC, Chen YT, Lin KC, Yang HW. Effects of mirror therapy on motor and sensory recovery in chronic stroke: A randomized controlled trial. Arch Phys Med Rehabil. 2013;94(6):1023-30.

11. Genius J, Roß S, Uhr S, Braun S, Rothgangel A, Verlag P. Practical protocol for stroke rehabilitation. Pain Pract. 2013;16(4):422-34.

12. Beyaert C, Vasa R, Frykberg GE. Gait post-stroke : Pathophysiology and rehabilitation strategies La marche après accident vasculaire cérébral : physiopathologie et stratégies de rééducation. Clin Neurophysiol. 2015;45(4-5):335-55.

13. Altschuler EL, Wisdom SB, Stone L, Foster C, Galasko D, Llewellyn DM, Ramachandran VS. Rehabilitation of hemiparesis after stroke with a mirror. Lancet. 1999 Jun 12;353(9169):2035-6.

14. Stevens JA, Stoykov ME. Using motor imagery in the rehabilitation of hemiparesis. Arch Phys Med Rehabil. 2003 Jul;84(7):1090-2.

15. Stevens JA, Stoykov ME. Simulation of bilateral movement training through mirror reflection: a case report demonstrating an occupational therapy technique for hemiparesis. Top Stroke Rehabil. 2004 Winter;11(1):59-66.

16. Sathian K, Greenspan Al, Wolf SL. Doing it with mirrors: a case study of a novel approach to neurorehabilitation. Neurorehabil Neural Repair. 2000;14(1):73-6.

17. Tyson S, Wilkinson J, Thomas N, Selles R, McCabe C, Tyrrell P, et al. Phase II pragmatic randomized controlled trial of patient-led therapies (mirror therapy and lower-limb exercises) during inpatient stroke rehabilitation. Neurorehabil Neural Repair. 2015 29(9):818-26.

18. Deconinck FJ, Smorenburg AR, Benham A, Ledebt A, Feltham MG, Savelsbergh GJ. Reflections on mirror therapy: a systematic review of the effect of mirror visual feedback on the brain. Neurorehabil Neural Repair. 2015 May;29(4):349-61.

19. Moher D, Schulz KF, Altman DG. The CONSORT statement: revised recommendations for improving the quality of reports of parallelgroup randomised trials. Lancet. 2001 Apr 14;357(9263):1191-4.

20. Chan AW, Tetzlaff JM, Altman DG, Laupacis A, Gøtzsche PC, et al. SPIRIT 2013 statement: defining standard protocol items for clinical trials. Ann Intern Med. 2013 Feb 5;158(3):200-7.

21. Meyer MJ, Pereira S, McClure A, Teasell R, Thind A, Koval J, Richardson M, Speechley M. A systematic review of studies reporting multivariable models to predict functional outcomes after post-stroke inpatient rehabilitation. Disabil Rehabil. 2015;37(15):1316-23.

22. Arya KN. Underlying neural mechanisms of mirror therapy: Implications for motor rehabilitation in stroke. Neurol India. 2016 Jan-Feb;64(1):38-44.

23. Flor $\mathrm{H}$, Diers M. Sensorimotor training and cortical reorganization. NeuroRehabilitation. 2009;25(1):19-27.

24. Sathian K. Mirror, mirror, move my manu! Neurorehabil Neural Repair. 2009 Mar-Apr;23(3):207-8.

25. Crosby LD, Marrocco S, Brown J, Patterson KK. A novel bilateral lower extremity mirror therapy intervention for individuals with stroke. Heliyon. 2016 Dec 7;2(12):e00208.

26. Lee HJ, Kim YM, Lee DK. The effects of action observation training and mirror therapy on gait and balance in stroke patients. J Phys Ther Sci. 2017 Mar;29(3):523-526. 